\title{
DEMONSTRATION OF THE APPLICATION OF SUPERPLASTIC FORMING USING INFRARED HEATING EMITTERS TO A PART OF STRUCTURE INCLUDING VARIOUS GEOMETRICAL SINGULARITIES
}

\author{
Elise LAMIC $(*)$, Nicolas FLEURISSON $(*)$, Fabien NAZARET $(*)$, Jean-Pierre BONNAFÉ $(* *)$, Ludovic ROPARS (**), Alexandre \\ $\operatorname{COLLOT}(* *)$
}

(*) Aurock - Albi, France (**) ArianeGroup - Les Mureaux

lamic@aurock.fr

ArianeGroup and Aurock led a feasibility study through the realization of a scale 1 TA6V demonstrator, using superplastic forming (SPF). ArianeGroup designed the demonstrator according to its knowledge of representative structures, comprising singularities: welds, stiffeners and areas with important thicknesses variations. Aurock performed first numerical simulations of the complete process, putting in evidence the various difficulties to be solved. Then, the demonstrator was physically carried out.

Once the demonstration was virtually obtained, each steps of the process were experimented: welding of thick plates with limited deformation, machining of flat panels, pre-forming by rolling and final SPF. For the SPF step, a heating cover and a reinforced refractory castable die were manufactured. Infrared emitters' position and heating power regulation laws were carefully defined, for the panel to be kept at the correct temperature until being formed.

The SPF step led to a successful demonstration of the representative structure. The experimental approach confirmed the process modelling predictability. Limited Scale 1 demonstration was necessary to ensure the process validity with real thicknesses and thickness variations, which are known to mask problems if scale reductions are used without precautions. This methodology can be transfer to a real structure only by tooling adaptations, without additional feasibility works.

\section{Introduction}

ArianeGroup develops and supplies innovative and competitive solutions for civil and military space launchers (Figure 1). ArianeGroup is lead contractor for Europe's Ariane 5 and Ariane 6 launcher families, as well as for the missiles of the French oceanic deterrent force. The group is a joint venture equally owned by Airbus and Safran, and employs approximately 9,000 highly qualified staff in France and Germany.
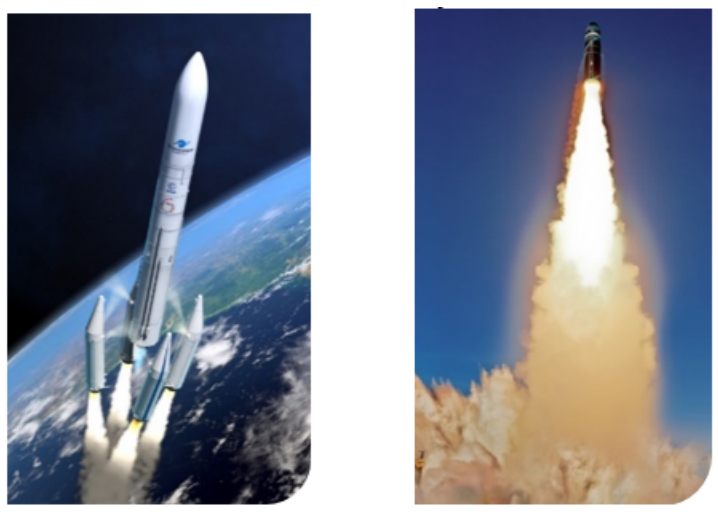

Figure 1: Examples of launch vehicles 
This unique know-how allows ArianeGroup to handle the entire lifecycle of its launch vehicles, from preliminary Research and Technology steps up to operations or dismantling. Most of the structures are made of aluminum alloys and high resistance/module composites but titanium alloys exhibits several interesting properties such as mechanical, thermal, forming and assembling properties that make this material inescapable. In order to validate the application of an improved forming process using superplastic capabilities of the material, a demonstrator including complex geometrical singularities were defined and tested in collaboration with Aurock.

Aurock is a SME with a strong expertise around the Superplastic Forming (SPF) process and process modelling. The company is specialized in three main activities: manufacturing of aerospace parts by SPF of titanium and aluminum alloys sheets, manufacturing of refractory castable tools and press components for high temperature applications and lastly specialized in metal forming processes simulation.

The principle of the SPF is to homogeneously heat a blank of a superplastic material at its superplasticity temperature (around $900^{\circ} \mathrm{C}$ for TA6V [1]) and to apply a monitored gas pressure vs. time to form the blank on a die at a controlled strain rate $\varepsilon^{-}$(classically $5.10^{-4} \mathrm{~s}^{-1}$ for TA6V [1]). In 2015, Aurock patented a new approach of SPF for titanium using localized infrared (IR) emitters heating instead of a global heating press [2]. The foremost interests of this new developed technique is to significantly enhance the heat efficiency (direct heating by radiations), the tooling management flexibility and to considerably reduce the cost of the process.

Together Aurock and ArianeGroup led a feasibility study of a limited scale 1 demonstrator using IR Superplastic forming after pre-forming step. This paper details the different stages realized to achieve the demonstration (Figure 2) : from the demonstrator design and manufacturing steps selection, to the complete forming of the part, going through important simulation phases for a de-risking approach with process parameters tuning.

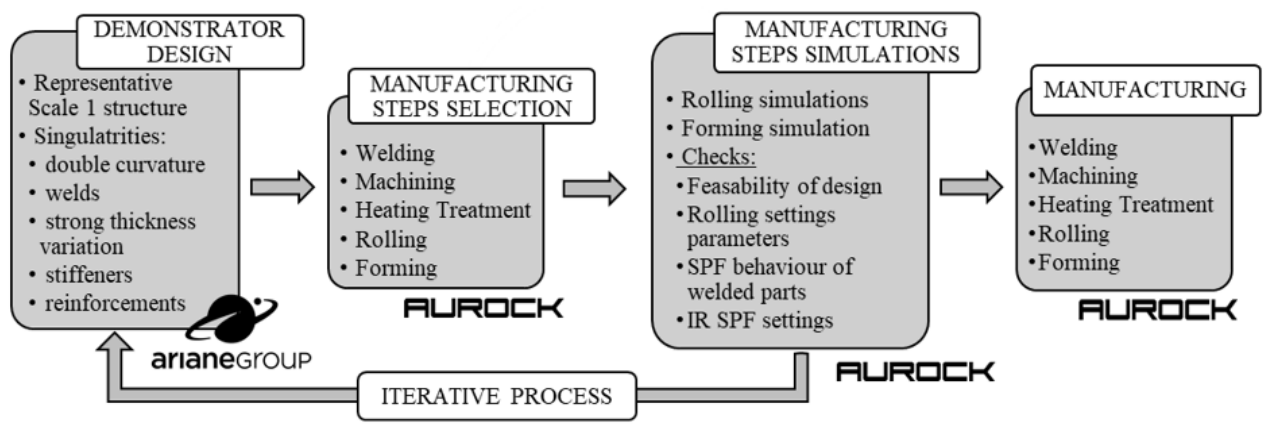

Figure 2: Overview of the feasibility study process followed - workshare between ArianeGroup and Aurock

\section{Forming process selection, demonstrator definition and de-risking approach with modelling}

\section{Selection of the forming process}

The major characteristics of launchers parts are the size of these parts, which drives to use appropriate machines and equipment and can limit or restrain the application of interesting technologies, and the low number of parts which has a direct impact on economical return on investment. In the case of classic superplastic forming, the size of a structure over 2 meters in diameter and several meters length would lead to the use of a very large press to include the mold and its heating device. All the interest of the infrared heating solution settled by Aurock [3] is to limit the heating to the part itself and to reduce strongly the size of the press, making this forming solution applicable and profitable. The main differences between a superplastic forming performed with a conventional SPF press and one with infrared emitters are summarized in the table 1 : 


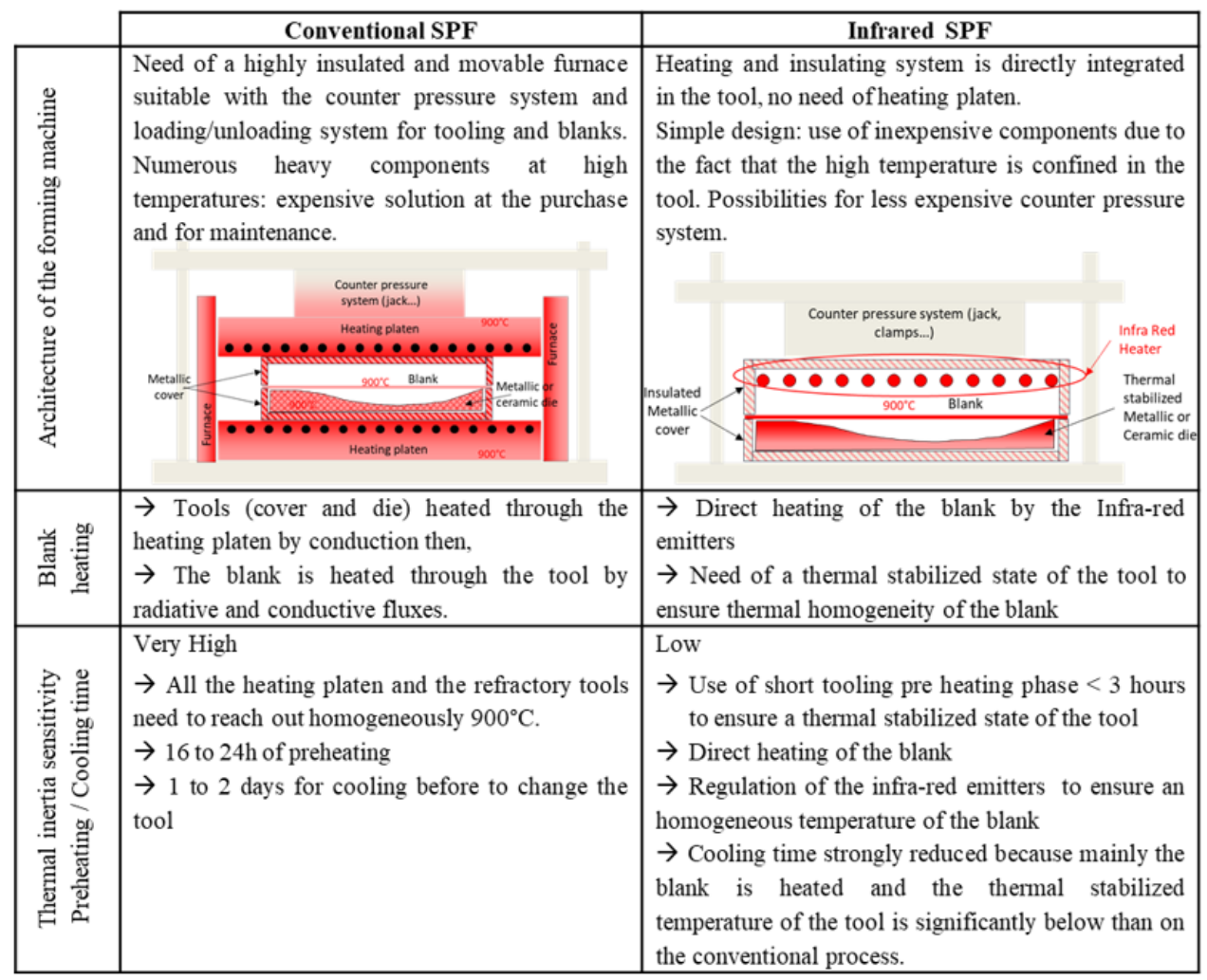

Table 1: Comparison between conventional SPF and Infra-Red SPF

Refractory castable reinforced by metallic fibers dies developed and patented by Aurock [4] for SPF application are particularly suitable for small manufacturing batches. In the frame of a demonstrator study, the solution of a ceramic die was selected due to a lower cost than a metallic one.

Choice of a configuration for the demonstrator

A launcher component is a structure made of aluminum or titanium alloy, with an axisymmetric shape with a simple or a double curvature, designed with or without stiffeners and/or local thick zones to reinforce the structure on specific places (propellant line supports or wall crossing, distancing rocket support).

The size of a launcher structure prevents us to perform a complete scale 1 demonstrator: solution was either to perform a complete structure demonstrator at reduced scale, or to perform a part of the structure at scale 1 . This second option was selected to avoid limitations and distorted conclusions given by a reduced scale configuration. The size of the demonstrator has been limited to be able to use the available press device at Aurock plant. A dummy part was defined cumulating the main difficulties: use of a titanium alloy not dedicated to superplastic behavior, thin skin with stiffeners and thick local reinforcement, double curvature with one large radius and a smaller one in the perpendicular direction.

The material is a classical titanium alloy TiAl6V4 in hot rolled and annealed conditions according to ABS 5125 and AIMS 03-18-001. This alloy is of standard quality due to the height of stiffeners: too thick to order SPF quality. Plates 2000x1000x20 $\mathrm{mm}^{3}$ were ordered. This size is sufficient to manufacture the demonstrator but will not be sufficient for the real size of a future structure, so that it could be necessary to weld rough plates to reach the correct size. We had then to know if such a weld on $20 \mathrm{~mm}$ thick could be achieved with a correct result, and 
what would be the behavior of such welds during superplastic forming, so a welded junction was added in the demonstrator (Figure 3).

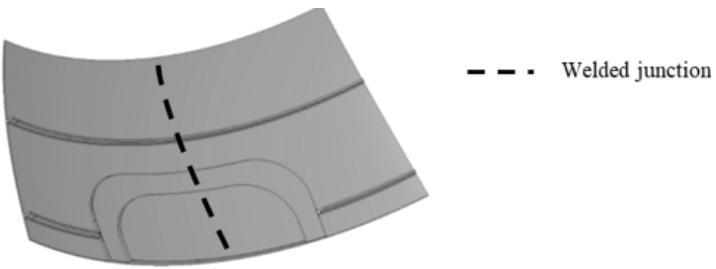

Figure 3: Design retained for the demonstrator

A preforming step before the final SPF was decided with an axisymmetric conical shape. The use of a conical rough shape leads to a more complex implementation due to edge effects but the result is more demonstrative.

\section{Simulations of the manufacturing steps - de-risking approach}

Due to the demonstrator design with a double curvature, stiffeners and local thick reinforcements with strong variations of thicknesses, a preforming step by cold rolling was decided prior final forming. The rolling step enables to obtain the main curvature with a conical preform, the double curvature is obtained thanks to the SPF step. Several simulations were performed for these two forming steps. The use of modelling is a powerful, fast and inexpensive method allowing industrialization de-risking and manufacturing parameters optimization. Models implementation requires know-how and suitable input data to obtain meaningful results. The simulations enabled to define the blank design, to validate the feasibility of the foreseen process and to launch the manufacturing steps with confidence.

The cold rolling simulations had the following objectives:

- To check the rolling passes lead to acceptable plastic strain on the stress concentration areas and no buckling of the stiffeners

- To set the rolling parameters: load levels to apply, number of rolling passes for avoiding high cumulative plastic strain and tooling definition to protect local reinforcements or stiffeners
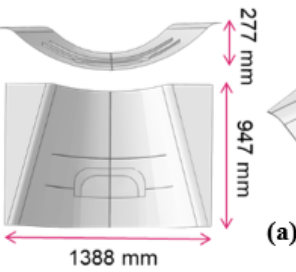

(a)
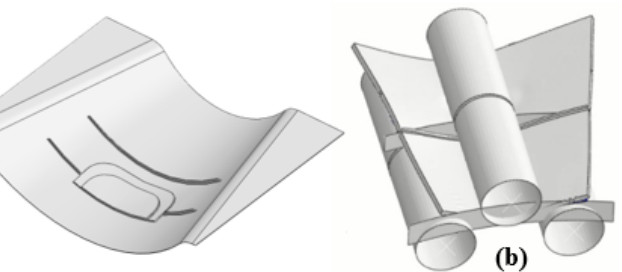

(b)

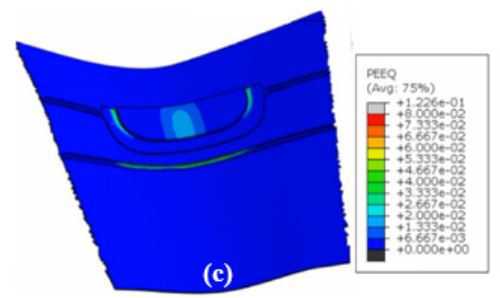

Figure 4: Cold rolling simulation step - (a) blank design at the end of the cold rolling step, (b) model, (c) Cumulative plastic strain at the end of the rolling step (several rolling passes)

For this simulation, an elasto-plastic constitutive law was used. The first design being locally too constraining, modifications of the junctions between stiffeners and thick local reinforcements were performed up to obtain satisfying results, leading to minor modifications with the optimization on junction radii. After this iterative loop, a final design satisfying all the previous described objectives was defined (Figure 4), rolling parameters with load level and number of passes were established and the SPF simulation step launched.

For the superplastic simulations the goals were to:

- check the feasibility of the forming even with the presence of stiffeners and local thick reinforcements : overall blank behavior check to 
meet the design requirements (no folds nor buckling of the stiffeners in the part area)

- check the level of pressure to apply for a full forming is compatible with the forming press capability,

- identify the pressure recipe to ensure a strain rate of the material at $5.10-4 \mathrm{~s}^{-1}$ during the forming phase,

- design the Infrared cover (position and power of the IR emitters)

- establish the power modulation recipes of the IR emitters during the forming to ensure an uniform blank temperature

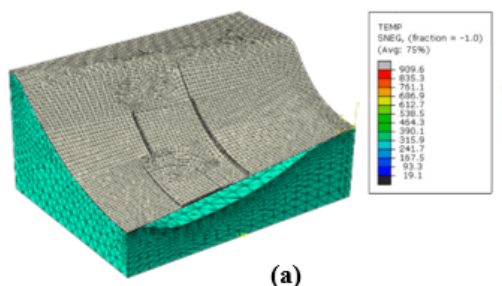

(a)
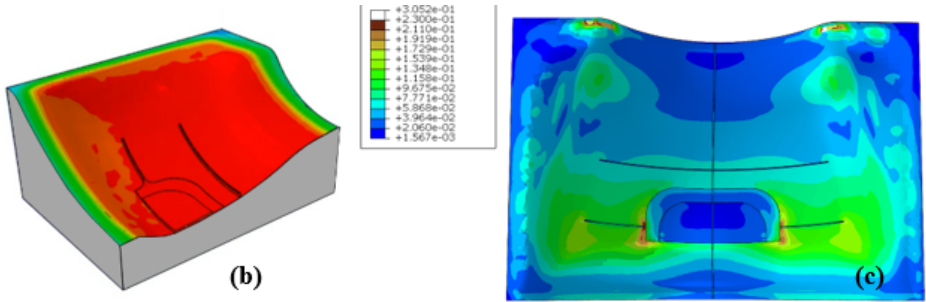

Figure 5: SPF simulations results, (a) model meshing, (b) thermal simulation enabling the IR cover design and modulation receipts definition of the emitters during forming, (c) mechanical simulation with equivalent creep strain results

A Norton-Hoff constitutive law describing the TA6V behavior at high temperature was used. After the tuning of the welded technique (described in Figure 5), a characterization step of the superplastic behavior of the welded junction was done.

According to the simulation results (Figure 5), the maximum creep strain obtained on the part was $21 \%$ with a maximum of $11 \%$ near the welded area. The global behavior of the blank was suitable to launch the manufacturing steps: two folds were identified but out of the part area, full forming is reached at a maximum of 6 bars with a pressure cycle of $42 \mathrm{~min}$ which is compatible with the selected press and no buckling of stiffeners was reported. All the simulations results were satisfying to launch the experimental demonstration with a high confidence level. All the experimental steps are presented in following chapters.

\section{Experimental demonstration}

\section{Thick plates welding and machining}

Preliminary tests with laser welding up to $12 \mathrm{~kW}$ showed that the maximum acceptable thickness in one welding pass was $13 \mathrm{~mm}$ which was not acceptable for our $20 \mathrm{~mm}$ thick panels. The first coupons were then obtained by two passes, one on each side. X-rays controls put in evidence porosities all along welded junction so this technique was not selected.

A hybrid technique was then tried combining laser and MIG welding. This approach significantly enhanced the outgazing and enabled to obtained healthy welded area without any defects after inspections (Figure 6). The hardness measures of the weld bead were also satisfactory.

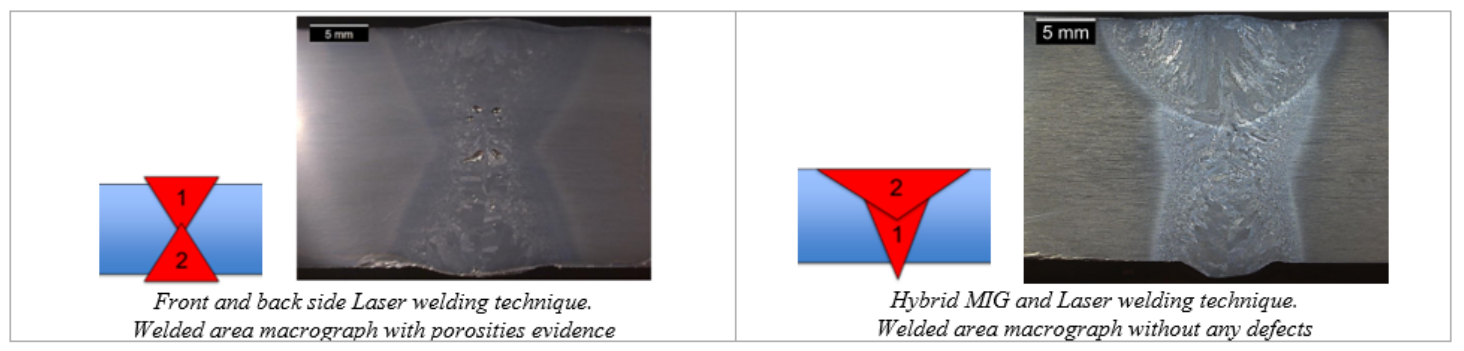

Figure 6: Experimental results of the welding technique tuning phase for the $20 \mathrm{~mm}$ thick TA6V panels junction 
This technique was then applied to the $20 \mathrm{~mm}$ thick panels. The removal of the weld bead leads to residual strain on the panel so a thermal treatment was then performed to ensure the flatness of the welded panels before machining. Machining step was then conducted: all the wanted design singularities, stiffeners, thick reinforcements with strong thickness variations were realized (Figure 7).
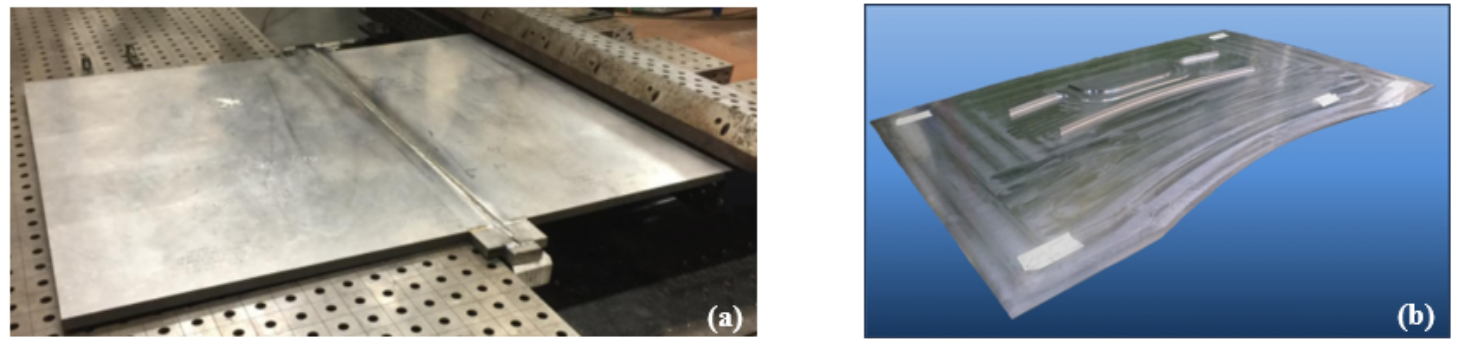

Figure 7: (a) Welded and (b) Machined panels

Preform achievement with conical rough shape

Due to strong thicknesses variations on the panels, specific elastomeric and metallic tools were designed for the rolling steps to prevent stiffeners crushing and to enable a suitable loads repartition. The main challenge of this step was to deal with the high titanium springback and the strong stiffness variations all along the different sections of the demonstrator. The outputs of the simulations presented in Figure 5 were highly useful to succeed in the calibration of this step.

The final setting was a hybrid solution combining successive roll passes and localized folds. Three set of parameters were established to obtain the conical preform (Figure 8), one for each specific area of the panel.

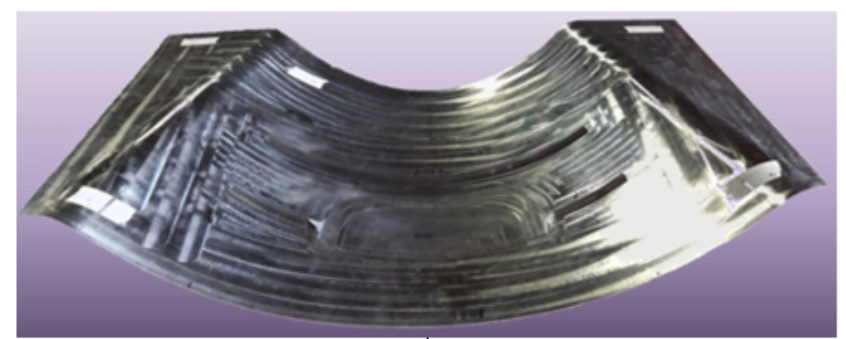

Figure 8: Conical preform of the demonstrator

Manufacturing of the ceramic die and its Infrared heating cover

In addition to economic considerations, usage of a ceramic die was decided with the following criterions:

- High compressive mechanical properties at elevated temperature

- Smooth state of surface as the blank will be in contact with the mold

- Thermal gradient resistance as only the die upper part is at high temperature

For this demonstrator, the sealing path enabling to reach the SPF maximum pressure is directly on the die without usage of an external casing to undertake the sealing loads. Due to the conical preform design, the sealing path has a non-planar progressive shape (Figure 9). 


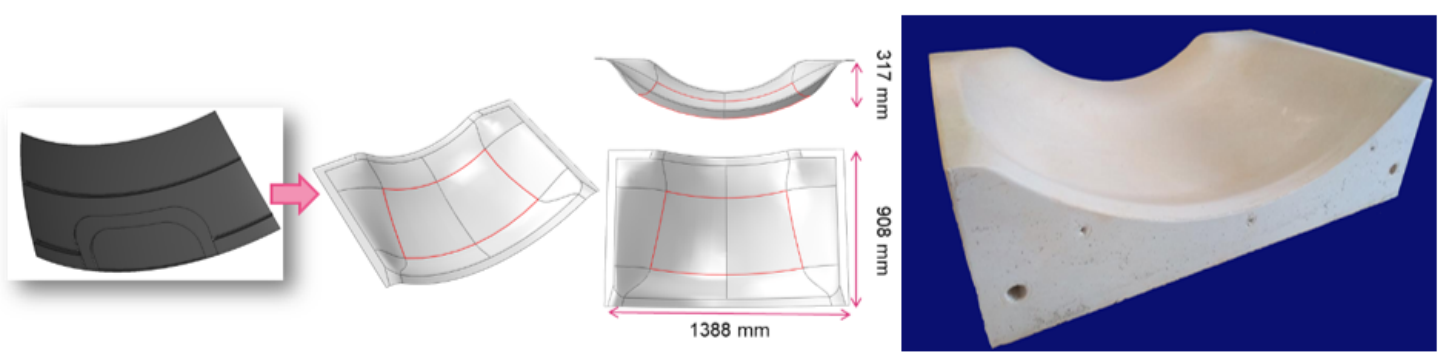

Figure 9: Design and Manufacturing of the reinforced refractory castable die

The cover architecture was defined with a network of 36 Infrared emitters from 2100 to $3400 \mathrm{~W}$ each. This design of the IR heating cover was made thanks to a numerical study to allow a homogeneous temperature on the entire blank surface to be reached during the heating step through individual power modulation (Figure 10).
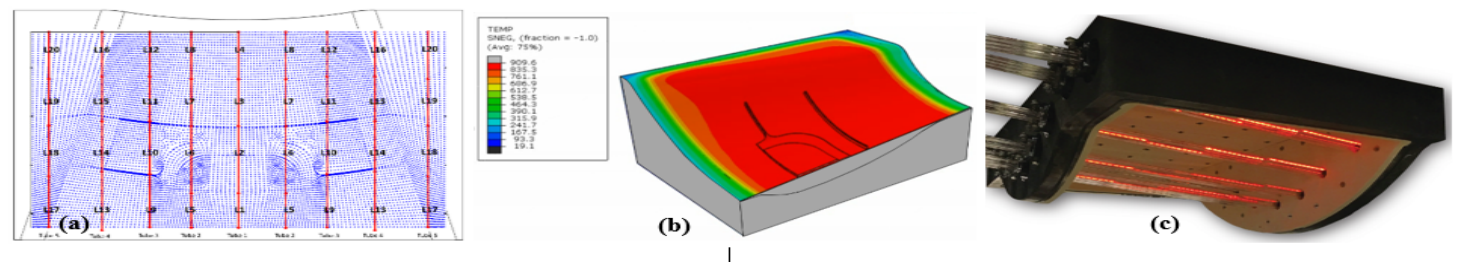

Figure 10: (a) Infrared cover architecture, (b) blank thermal state at the end of the heating phase, (c) IR cover

The key point to succeed in an IR SPF is the emitters' individual power recipe definition. These recipes are defined via numerical optimization to reach the most homogeneous blank temperature with minimal thermal gradient over the surface and through the thickness before to start the pressure forming cycle. The presence of strong thicknesses variations and stiffeners made this particularly challenging. A long heating phase was privileged and the selected cover architecture enables to reach homogeneously the targeted temperature at $870^{\circ} \mathrm{C}$ with $+/-35^{\circ} \mathrm{C}$ in approximatively $2 \mathrm{~h} 15 \mathrm{~min}$ without any local overheating.

\section{Demonstrator forming}

The assembly of the die and its infrared cover was mounted on the Aurock 400T press (Figure 11). Prior forming tests, the upper surface of the die and both sides of the preformed demonstrator blank were coated with Boron Nitride lubricant to ease the forming and give a barrier to oxidation. The preformed blank was put on the cold ceramic die, preheating started during $2 \mathrm{~h} 20 \mathrm{~min}$, sealing was made when the blank homogeneously reached $870^{\circ} \mathrm{C}$ at the end of the heating phase and finally the pressure forming cycle started, reaching up to 6 bars after 40 minutes. Live monitoring and control of the forming and comparison with simulation results was performed using pressure sensors and 15 thermocouples placed in the die and on the blank. 


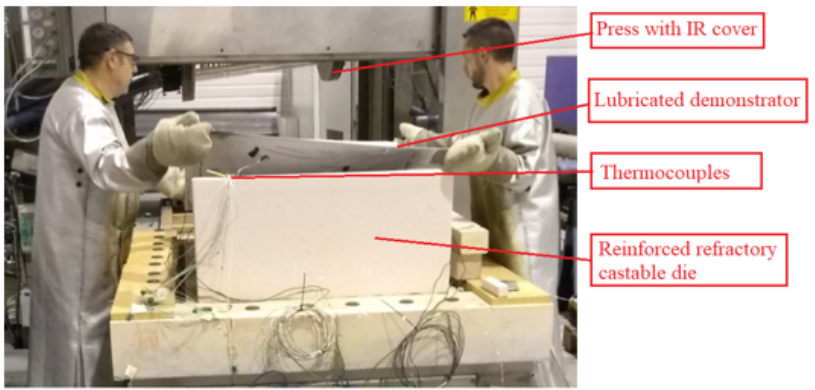

Figure 11: View of the assembly under the Aurock 400T forming press

Results

A visual inspection carried out post forming indicated the full forming of the demonstrator without any defects (Figure 12). Two folds were obtained as predicted out of the part area. The ultrasonic thickness controls show an excellent correlation with the simulations predictions (gap lower than 5\%). A tridimensional laser check before part cutting confirmed that the post-formed part geometry is within the requested tolerances.

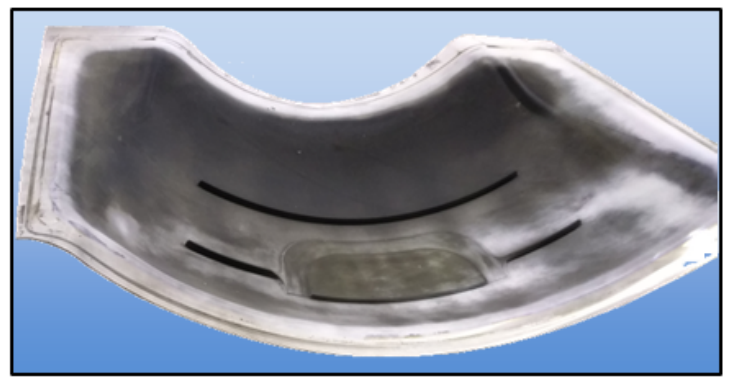

Figure 12: Infrared superplastic formed demonstrator before part cutting and chemical machining

\section{Conclusions and outlooks}

A feasibility study through the realization of a scale 1 representative demonstrator designed by ArianeGroup, using Infrared superplastic forming was successfully achieved by Aurock. The use of a de-risking step by step approach supported by FE process modelling pre-studies strongly contributed to this success. Difficulties have been highlighted and solved before to launch the demonstrator physical realization. Once the demonstration was virtually obtained, each steps of the process were successively experimented: welding of thick plates with limited deformation, machining of flat panels, pre-forming by rolling and final superplastic forming with Infrared heaters. The experimental approach and controls on the demonstrator confirmed the process modelling predictability and the robustness of the IR forming process. Thus, a simulation tool is available for the future to reduce the experimental loops and to adapt potential definition variations of a real structure.

\section{Acknowledgements}

The authors acknowledge Institut Maupertuis for the welding step tuning and achievement and Chaudronnerie Brilla for the rolling step manufacturing. Their respective skills contributed to the success of this challenging project. 


\section{References}

[1] G.Giuliano, Superplastic forming of advanced metallic materials - methods and applications, ISBN 978-1-84569-753-2 (2011), p.12.

[2] F.Nazaret, Method of controlling a superplastic forming machine and correspondent machine, FR patent number FR3034690A1, 2015 WO 2016/162642 A1, 2016.

[3] D.Mauduit, M.LeFournier, K.Grondin, T.Pottier, Y.Le-Maoult, Industrial applications of the superplastic forming by using Infrared heaters, International Conference on the Technology of Plasticity, ICTP 2017, Cambridge, UK.

[4] F.Nazaret, O.Barrau, G.Bernhart, T.Cutard, Innovative SPF toolings based on reinforced refractory concretes, ICSAM2009 International Conference on Superplasticity in Advanced Materials (Seattle) Invited lecture 2009. 